\title{
Gray Matter Microstructural Abnormalities and Working Memory Deficits in Individuals with Schizophrenia
}

\author{
HyunJung Kim¹, Seung-Hyun Shon², Sung Woo Joo ${ }^{3}$, Woon Yoon², \\ Jang-Han Lee ${ }^{4}$, Ji-Won Hur ${ }^{4}$, and JungSun Lee ${ }^{2} \bowtie$ \\ 1'Department of Clinical \& Counseling Psychology, Graduate School of Psychological Service, Chung-Ang University, Seoul, Republic of Korea \\ ${ }^{2}$ Department of Psychiatry, University of Ulsan College of Medicine, Asan Medical Center, Seoul, Republic of Korea \\ ${ }^{3}$ Republic of Korea Marine Corps Education and Training Center, Pohang, Republic of Korea \\ ${ }^{4}$ Department of Psychology, Chung-Ang University, Seoul, Republic of Korea
}

Objective Working memory impairments serve as prognostic factors for patients with schizophrenia. Working memory deficits are mainly associated with gray matter (GM) thickness and volume. We investigated the association between GM diffusivity and working memory in controls and individuals with schizophrenia.

Methods $\mathrm{T} 1$ and diffusion tensor images of the brain, working memory task (letter number sequencing) scores, and the demographic data of 90 individuals with schizophrenia and 97 controls were collected from the SchizConnect database. T1 images were parcellated into the 68 GM Regions of Interest (ROI). Axial Diffusivity (AD), Fractional Anisotropy (FA), Radial Diffusivity (RD), and Trace (TR) were calculated for each of the ROIs.

Results Compared to the controls, schizophrenia group showed significantly increased AD, RD, and TR in specific regions on the frontal, temporal, and anterior cingulate area. Moreover, working memory was negatively correlated with $\mathrm{AD}, \mathrm{RD}$, and TR in the lateral orbitofrontal, superior temporal, inferior temporal, and rostral anterior cingulate area on left hemisphere in the individuals with schizophrenia.

Conclusion These results demonstrated GM microstructural abnormalities in the frontal, temporal, and anterior cingulate regions of individuals with schizophrenia. Furthermore, these regional GM microstructural abnormalities suggest a neuropathological basis for the working memory deficits observed clinically in individuals with schizophrenia.

Psychiatry Investig 2019;16(3):234-243

Key Words Diffusion tensor imaging, Gray matter, Working memory, Schizophrenia, Diffusivity.

\section{INTRODUCTION}

Working memory impairments are considered central in schizophrenia. ${ }^{1}$ Because working memory impairments are one of the most enduring symptoms, they are also strong predictors of poor clinical outcome in individuals with schizophrenia. ${ }^{2}$ Thus, individuals with schizophrenia often experience problems in their daily activities, such as difficulties remembering phone numbers or engaging in mental arithmetic. The causes behind working memory abnormality have been pre-

Received: May 30, 2018 Revised: September 12, 2018

Accepted: October 14, 2018

$\triangle$ Correspondence: JungSun Lee, MD, PhD

Department of Psychiatry, University of Ulsan College of Medicine, Asan Medical Center, 88 Olympic-ro 43-gil, Songpa-gu, Seoul 05505, Republic of Korea

Tel: +82-2-3010-3422, Fax: +82-2-485-8381, E-mail: js_lee@amc.seoul.kr

(c) This is an Open Access article distributed under the terms of the Creative Commons Attribution Non-Commercial License (http://creativecommons.org/licenses/bync/4.0) which permits unrestricted non-commercial use, distribution, and reproduction in any medium, provided the original work is properly cited. dominantly reported through the volume and thickness of gray matter (GM). ${ }^{3}$ Such studies, however, have only focused on the macrostructural differences and thus have not captured microstructural abnormalities. Diffusion tensor imaging (DTI) studies are useful to study microstructural abnormalities through white matter (WM), and WM-DTI studies have reported that the abnormalities of working memory are associated with $\mathrm{WM}^{4-6}$ and depend on myelinated axonal fibers. ${ }^{7-10}$

DTI studies have typically investigated WM microstructure by examining the water molecule's diffusivity in the brain. Compared to WM-DTI studies, only a few studies have investigated GM microstructure in the frontal and/or temporal cortex ${ }^{11-14}$ entorhinal cortex,${ }^{15}$ and parahippocampal, insula, and anterior cingulate region ${ }^{16}$ of individuals with schizophrenia. These studies were conducted using diffusivity in GM, such as axial diffusivity $(\mathrm{AD})$, radial diffusivity $(\mathrm{RD})$, fractional anisotropy (FA), trace (TR) or mean diffusivity (MD). As a result, positive GM-DTI findings in schizophrenia have been report- 
ed: 1) increased MD was shown, bilaterally, in the superior temporal gyrus (STG) GM and the left STG WM. MD in left STG WM demonstrated the association with attentional impairments and auditory hallucinations in schizophrenia; ${ }^{12} 2$ ) increased TR in the bank of the superior temporal sulcus of left hemisphere and the inferior temporal, the middle temporal, the inferior parietal gyri of right hemisphere in schizophrenia. ${ }^{11}$ TR in the middle temporal gyrus and the inferior temporal gyrus of right hemisphere had also negative correlation with social cognition in schizophrenia; ${ }^{11} 3$ ) increased $\mathrm{AD}$ and $\mathrm{RD}$ were shown in the temporal and prefrontal cortices. $\mathrm{AD}$ and $\mathrm{RD}$ were associate with positive symptom of schizophrenia; ${ }^{13} 4$ ) there is elevated diffusivity in the frontotemporal regions on schizophrenia; ${ }^{14} 5$ ) increased MD was showed in the insula, anterior cingulate gyrus, and parahippocampal gyrus of right hemisphere in schizophrenia. ${ }^{16}$

The diffusion anisotropy in GM cortex has been observed in the animal, ${ }^{17-20}$ and the human. ${ }^{21-26}$ Radial diffusion anisotropy has also been demonstrated in the animal, ${ }^{27,28}$ and the human. ${ }^{28-35}$ The principal diffusion direction shows apparent structure within GM, running nearly perpendicular to the cortical surface. ${ }^{30}$ And the principal diffusion component's orientation tends to be more within a plane parallel to the cortical surface ${ }^{36}$ Although $\mathrm{RD}$ and $\mathrm{AD}$ have been focused on the evaluation of axon integrity in WM, evidence demonstrate that the direction of diffusion in GM occurs both radial (perpendicular) and axial (parallel) to the surface of the cortex..$^{28,30}$ Thus, $\mathrm{RD}$ and $\mathrm{AD}$ in GM may reflect pyramidal cell's microstructural integrity. ${ }^{37}$ Investigating this phenomenon, a detailed study suggests that parallel and radial structure are believed to exist, and actually be a microscopic marker that is able to distinguish brain areas. $^{28}$

The cause of lower cases of GM-DTI than WM-DTI is that GM studies have typically investigated volume and thickness reductions in schizophrenia. Interestingly, in individuals with schizophrenia, the decreased GM thickness and volume have been shown by reduced presence of membranes, axon terminals, dendrites, and dendritic spines. ${ }^{38-40}$ These findings suggest that the intrinsic microstructure of GM, rather than its volume and thickness, is more important for understanding the neuropathological basis of GM abnormalities in schizophrenia.

Despite the advantages of the use of DTI to study GM microstructure, previous DTI studies have focused on WM, and the few GM-DTI studies have focused on specific GM regions or they involved small sample sizes. According to previous schizophrenia studies, there are more than 50 regional deficits, such as in frontal, temporal, parietal, and cingulate areas and in the deficits, ${ }^{41}$ and a larger group will give us better understanding the feature of these microscopic alternations and their associations with clinical profiles and symptoms. ${ }^{42}$
Therefore, it is necessary to study whole GM regions with larger samples using sufficient data sources. The SchizConnect database (www.schizconnect.org) shows Significant possibilities to overcome the barriers related to the creation of large data sets to elevate statistical power. ${ }^{43}$ Consequently, our study used the SchizConnect database to examine entire GM regions in a larger sample.

This study investigated the hypothesis that GM diffusivity in regions of interest (ROIs) would differ between individuals with schizophrenia and control subjects and that GM diffusivity and working memory would correlate significantly.

\section{METHODS}

\section{Data sources}

This study used the demographic data, brain images (T1 and DTI), and working memory performance test data of 229 cases included in the SchizConnect database used in the Center for Biomedical Research Excellence (COBRE) and Neuromorphometry by Computer Algorithm Chicago (NMorphCH) studies. $^{44}$

\section{Study participants}

Of the 229 cases, 42 were excluded for the following reasons: missing cognitive function test values $(n=22)$, diagnoses of schizoaffective disorder $(\mathrm{n}=10)$, and poor brain imaging quality $(n=10)$. The remaining 187 cases were divided into two groups: patients with schizophrenia $(n=90)$ and control subjects $(n=97)$. The age range of the participants was 20 to 66 years. This study was approved by the Institutional Review Board of Asan Medical Center (IRB File No. S2017-1996-0001).

\section{Diffusivity}

T1 and DTI neuroimages were used. In the COBRE database, the T1 scan parameters were as follows: repetition time $(\mathrm{TR})=2,530 \mathrm{~ms}$, echo time $(\mathrm{TE})=1.64 \mathrm{~ms}$, flip $=0^{\circ}$, matrix $=$ $256 \times 256$, slices $=192$, and slice thickness $=1 \mathrm{~mm}$. The DTI scan parameters were as follows: $\mathrm{TR}=9,000 \mathrm{~ms}, \mathrm{TE}=84 \mathrm{~ms}$, flip $=0^{\circ}$, matrix $=256 \times 256$, slices $=72$, and slice thickness $=2$ $\mathrm{mm}$. In the NMorphCH database, the $\mathrm{T} 1$ scan parameters were as follows: $\mathrm{TR}=2,400 \mathrm{~ms}, \mathrm{TE}=3.16 \mathrm{~ms}$, flip $=8^{\circ}$, matrix $=$ $256 \times 256$, slices $=176$, and slice thickness $=1 \mathrm{~mm}$. The DTI scan parameters were as follows: $\mathrm{TR}=8,000 \mathrm{~ms}, \mathrm{TE}=86 \mathrm{~ms}$, flip $=90^{\circ}$, matrix $=896 \times 896$, slices $=35$, and slice thickness $=2 \mathrm{~mm}$.

Motion and eddy current-induced distortions were corrected with the affine registration of all gradient volumes with the first $b=0$ volume using the FMRIB Software Library (FLIRT: FSL, version 6.0.0; http://fsl.fmrib.ox.ac.uk/fsl/fslwiki/). ${ }^{45,46}$ Our calculations of diffusivities were conducted using eigenvalues $(\lambda 1, \lambda 2$, and $\lambda 3)$. FA was automatically calculated in 
dtifit in FSL. The following additional diffusivity values were obtained for the whole brain of each subject using eigenvalues in fslmaths in FSL: 1) AD $(\lambda 1), 2) \mathrm{RD}[(\lambda 2+\lambda 3) / 2]$, and 3) $\operatorname{TR}(\lambda 1+\lambda 2+\lambda 3)$.

The $\mathrm{T} 1$ images were parcellated into discrete anatomical regions using the Desikan-Killiany atlas of FreeSurfer (version 6.0; http://surfer.nmr.mgh.harvard.edu) ${ }^{47}$ and all parcellated ROIs of the GM ( $n=68)$ (Supplementary Table 1 in the online-only Data Supplement) were used in the subsequent analyses. The $\mathrm{T} 1$ images were registered to $\mathrm{a} b=0$ baseline DTI image using flirt in FSL. The parcellated labels were then transformed into DTI using the same registration transformation. Next, we calculated the diffusivities (AD, FA, RD, and TR) for each transformed ROI of each subject. Z-scores were calculated using the means and standard deviations of each ROI of the control group in each project.

\section{Symptoms}

Patients' psychiatric symptoms were evaluated by a psychiatrist using the Positive and Negative Syndrome Scale (PANSS) in COBRE and The Scale for the Assessment of Positive Symptoms (SAPS), the Scale for the Assessment of Negative Symptoms (SANS) in NMorphCH studies.

\section{Working memory}

We used the scaled scores from the Letter Number Sequencing (LNS) test. The LNS test assesses the subject's ability to manipulate and rearrange verbal representations in working memory. The LNS test used in this study was included in the following different test batteries in the COBRE and NmorphCH projects: "Measurement and Treatment Research to Improve Cognition in Schizophrenia Consensus Cognitive Battery" ${ }^{48,49}$ and "Wechsler Memory Scale Third Edition" ${ }^{50}$ respectively. Thus, in this study, the scaled scores on the LNS test were converted into Z-scores for each project.

\section{Statistical analysis}

For the demographic data, t-tests were conducted to investigate differences in age, illness duration, and LNS scores between the groups. Chi-squared tests were conducted to examine differences in the sex ratios between the groups.

Next, statistical analyses controlling for sex and age were conducted. First, an analysis of covariance was conducted to test for significant differences in diffusivity (AD, FA, TR, and $\mathrm{RD})$ in the 68 ROIs between the control and patient groups. Due to the multiple comparisons of diffusivity in the 68 ROIs, the significance threshold was adjusted using the false discovery rate $(\mathrm{q}<0.05)$. Second, Pearson partial correlation analyses were conducted on the LNS scores and ROI diffusivities showing significant differences between the groups. All statistical analyses were performed using $\mathrm{R}$ (R Foundation for Statistical Computing, Vienna, Austria). ${ }^{51}$

\section{RESULTS}

\section{Demographic data}

The demographic and clinical information is presented in Table 1. The final data set included 187 subjects (97 controls

Table 1. Demographic and clinical data on the subjects

\begin{tabular}{|c|c|c|c|c|c|c|c|}
\hline & \multicolumn{2}{|c|}{ Control $(\mathrm{N}=97)$} & \multicolumn{2}{|c|}{ Schizophrenia $(\mathrm{N}=90)$} & \multirow{2}{*}{$\operatorname{tor} \chi^{2}$} & \multirow{2}{*}{ df } & \multirow{2}{*}{$\mathrm{p}$} \\
\hline & Mean & $\mathrm{SD}$ & Mean & $\mathrm{SD}$ & & & \\
\hline Age & 36.74 & 11.56 & 36.94 & 11.44 & -0.12 & 184.23 & 0.90 \\
\hline \multicolumn{8}{|l|}{ Gender $(\mathrm{N})$} \\
\hline Male & 72 & & 65 & & 0.18 & 1 & 0.66 \\
\hline Female & 25 & & 25 & & & & \\
\hline Duration of illness in COBRE (years) & & & 11.33 & 13.53 & & & \\
\hline \multicolumn{8}{|l|}{ PANSS in COBRE } \\
\hline Total score & & & 59.54 & 19.89 & & & \\
\hline Positive score & & & 15.24 & 5.25 & & & \\
\hline Negative score & & & 14.8 & 5.45 & & & \\
\hline General score & & & 29.5 & 9.19 & & & \\
\hline SAPS in NMorphCH & & & 42 & 34.39 & & & \\
\hline SANS in NMorphCH & & & 53.31 & 26.27 & & & \\
\hline LNS & -0.02 & 1.01 & -0.99 & 1.26 & 5.82 & 170.65 & $<0.001$ \\
\hline
\end{tabular}

SD: standard deviation, df: degrees of freedom, COBRE: center for biomedical research excellence, LNS: letter number sequencing, PANSS: positive and negative syndrome scale, SAPS: the scale for the assessment of positive symptoms, SANS: the scale for the assessment of negative symptoms 
and 90 patients). The age of the groups did not differ significantly [mean \pm standard deviation (SD): controls, 36.74 \pm 11.56 years; schizophrenia, $36.94 \pm 11.44$ years; $\mathrm{df}=184.23$, $\mathrm{t}=-0.1201$, $\mathrm{p}=0.90]$. The percentage of men in the control group was $74.2 \%$, higher than that in the schizophrenia group $(72.2 \%)$, but this difference was not statistically significant $\left(\chi^{2}=0.18\right.$, $\mathrm{df}=1, \mathrm{p}=0.66$ ). The mean duration of illness was $11.33 \pm 13.53$ years in the individuals with schizophrenia in COBRE. The LNS scores differed significantly between the control and schizophrenia groups (controls: $-0.02 \pm 1.01$; schizophrenia: $-0.99 \pm 1.26 ; \mathrm{df}=170.65, \mathrm{t}=5.82, \mathrm{p}<0.001)$. PANSS total score was $59.54 \pm 19.89$ (positive score: $15.24 \pm 5.25$; negative score: 14.8 \pm 5.45 ; general score $29.5 \pm 9.19$ ) in COBRE. SAPS total score was $42 \pm 34.39$ and SANS total score was $53.31 \pm 26.27$ in NMorphCH.

\section{Comparisons of GM diffusivity}

The diffusivity results are presented in Table 2 . All p values were corrected by the false discovery rate. FA values did not differ significantly between the two groups (FDR-corrected $\mathrm{p}>0.01)$.

Regarding AD, the schizophrenia group exhibited significantly increased $\mathrm{AD}$ values compared with those of the control group in the following 3 ROIs: 1) left medial orbitofrontal cortex [mean $\pm S D$ : control, $0.04 \pm 1.02$; schizophrenia, $0.61 \pm$ $1.15 ; \mathrm{F}(1,183)=12.71, \mathrm{p}<0.001], 2)$ left pars opercularis [control, $0.07 \pm 1$; schizophrenia, $0.57 \pm 1.03 ; \mathrm{F}(1,183)=11.68$, $\mathrm{p}<0.001$ ], and 3) left STG [control, 0.08 \pm 0.98 ; schizophrenia, $0.54 \pm 1.1 ; \mathrm{F}(1,183)=9.66, \mathrm{p}=0.002]$.

Regarding $\mathrm{RD}$, the schizophrenia group exhibited significantly increased $\mathrm{RD}$ values compared with those of the control group in the following 14 ROIs: 1) left bank of the superior

Table 2. Comparison of diffusivity values in the ROls with significant differences between the groups

\begin{tabular}{|c|c|c|c|c|c|c|}
\hline & \multirow{2}{*}{ ROI } & \multicolumn{2}{|c|}{ Control } & \multicolumn{2}{|c|}{ Schizophrenia } & \multirow{2}{*}{$\mathrm{F}$} \\
\hline & & M & SD & M & $\mathrm{SD}$ & \\
\hline \multirow[t]{3}{*}{$\mathrm{AD}$} & Left medial orbitofrontal cortex & 0.04 & 1.02 & 0.61 & 1.15 & $12.71^{\dagger}$ \\
\hline & Left pars opercularis & 0.07 & 1 & 0.57 & 1.03 & $11.68^{*}$ \\
\hline & Left superior temporal gyrus & 0.08 & 0.98 & 0.54 & 1.1 & $9.66^{*}$ \\
\hline \multirow[t]{14}{*}{$\mathrm{RD}$} & Left bank of the superior temporal sulcus & 0.08 & 0.99 & 0.5 & 0.98 & $8.3^{*}$ \\
\hline & Left inferior temporal gyrus & 0.06 & 0.99 & 0.53 & 1.09 & $9.61^{*}$ \\
\hline & Left lateral orbitofrontal cortex & 0.03 & 1.04 & 0.63 & 1.06 & $15.91^{\dagger}$ \\
\hline & Left medial orbitofrontal cortex & 0.01 & 1.03 & 0.61 & 1.2 & $13.36^{\dagger}$ \\
\hline & Left middle temporal gyrus & 0.01 & 1.04 & 0.59 & 1.02 & $16.59^{\dagger}$ \\
\hline & Left pars opercularis & 0.04 & 1.02 & 0.71 & 1.1 & $20.10^{\dagger}$ \\
\hline & Left rostral anterior cingulate cortex & 0.03 & 1.04 & 0.48 & 1.09 & $8.57^{*}$ \\
\hline & Left rostral middle frontal gyrus & 0.03 & 1.02 & 0.46 & 0.86 & $10.39 *$ \\
\hline & Left superior temporal gyrus & 0.04 & 1.03 & 0.59 & 0.98 & $15.38^{\dagger}$ \\
\hline & Left temporal pole & 0.01 & 1.01 & 0.49 & 1.23 & $8.24^{*}$ \\
\hline & Right isthmus of the cingulate gyrus & 0.06 & 1.01 & 0.47 & 1.1 & $6.90^{*}$ \\
\hline & Right lateral orbitofrontal cortex & 0.03 & 1.02 & 0.47 & 1.19 & $7.20^{*}$ \\
\hline & Right pars opercularis & 0.05 & 1.03 & 0.49 & 1.29 & $7.18^{*}$ \\
\hline & Right frontal pole & 0.01 & 1.03 & 0.47 & 1.2 & $8.84^{*}$ \\
\hline \multirow[t]{9}{*}{ TR } & Left lateral orbitofrontal cortex & 0.03 & 1.04 & 0.56 & 1.05 & $12.03^{*}$ \\
\hline & Left medial orbitofrontal cortex & 0.02 & 1.02 & 0.63 & 1.2 & $13.95^{\dagger}$ \\
\hline & Left middle temporal gyrus & 0.02 & 1.03 & 0.58 & 1.06 & $14.82^{\dagger}$ \\
\hline & Left pars opercularis & 0.05 & 1.01 & 0.68 & 1.1 & $17.45^{\dagger}$ \\
\hline & Left rostral anterior cingulate cortex & 0.04 & 1.04 & 0.48 & 1.09 & $8.36^{*}$ \\
\hline & Left rostral middle frontal gyrus & 0.04 & 1.01 & 0.45 & 0.89 & $9.11^{*}$ \\
\hline & Left superior temporal gyrus & 0.06 & 1.01 & 0.59 & 1.02 & $14.27^{\dagger}$ \\
\hline & Left temporal pole & 0.01 & 1.01 & 0.47 & 1.22 & $7.80^{*}$ \\
\hline & Right frontal pole & 0.01 & 1.03 & 0.5 & 1.23 & $9.15^{*}$ \\
\hline
\end{tabular}

${ }^{*} \mathrm{p}<0.01,{ }^{\dagger} \mathrm{p}<0.001$. ROI: region of interest, SD: standard deviation, df: degrees of freedom, AD: axial diffusivity, RD: radial diffusivity, TR: trace 
temporal sulcus [mean \pm SD: control, $0.08 \pm 0.99$; schizophrenia, $0.5 \pm 0.98 ; \mathrm{F}(1,183)=8.3, \mathrm{p}=0.004]$, 2) left inferior temporal gyrus [control, $0.08 \pm 0.99 ;$ schizophrenia, $0.5 \pm 0.98 ; \mathrm{F}(1$, $183)=9.61, \mathrm{p}=0.002], 3)$ left lateral orbitofrontal cortex [control, 0.03 \pm 1.04 ; schizophrenia, $0.63 \pm 1.06 ; \mathrm{F}(1,183)=15.91$, $\mathrm{p}<0.001]$, 4) left medial orbitofrontal cortex [control, $0.01 \pm$ 1.03; schizophrenia, $0.61 \pm 1.2 ; \mathrm{F}(1,183)=13.36, \mathrm{p}<0.001]$, 5) left middle temporal gyrus [control, $0.01 \pm 1.04$; schizophrenia, $0.59 \pm 1.02 ; \mathrm{F}(1,183)=16.59, \mathrm{p}<0.001], 6)$ left pars opercularis [control, $0.04 \pm 1.02$; schizophrenia, $0.71 \pm 1.1 ; \mathrm{F}(1$, $183)=20.1, \mathrm{p}<0.001], 7)$ left rostral anterior cingulate cortex [control, 0.03 \pm 1.04; schizophrenia, $0.48 \pm 1.09 ; \mathrm{F}(1,183)=8.57$, $\mathrm{p}=0.003$ ], 8) rostral middle frontal gyrus [control, $0.03 \pm 1.02$; schizophrenia, $0.46 \pm 0.86 ; \mathrm{F}(1,183)=10.39, \mathrm{p}=0.001]$, 9) left STG [control, 0.04 \pm 1.03 ; schizophrenia, $0.59 \pm 0.98 ; \mathrm{F}(1,183)=$ $15.38, \mathrm{p}<0.001$ ] , 10) left temporal pole [control, $0.01 \pm 1.01$; schizophrenia, $0.49 \pm 1.23 ; \mathrm{F}(1,183)=8.24, \mathrm{p}=0.004]$, 11) right isthmus cingulate gyrus [control, $0.06 \pm 1.01$; schizophrenia, $0.47 \pm 1.1 ; \mathrm{F}(1,183)=6.9, \mathrm{p}=0.009], 12)$ right lateral orbitofrontal cortex [control, $0.03 \pm 1.02$; schizophrenia, $0.47 \pm 1.19 ; \mathrm{F}(1$, $183)=7.2, \mathrm{p}=0.008], 13)$ right pars opercularis [control, $0.05 \pm$ 1.03; schizophrenia, $0.49 \pm 1.29 ; \mathrm{F}(1,183)=7.18, \mathrm{p}=0.008$ ], and 14 ) right frontal pole [control, $0.01 \pm 1.03$; schizophrenia, $0.47 \pm$ 1.2; $\mathrm{F}(1,183)=8.84, \mathrm{p}=0.003$ ].

Regarding TR, the schizophrenia group exhibited significantly increased TR values compared with those of the control group in the following 9 ROIs: 1) left lateral orbitofrontal cortex [mean \pm SD: control, $0.03 \pm 1.04$; schizophrenia, $0.56 \pm$ 1.05; $\mathrm{F}(1,183)=12.03, \mathrm{p}<0.001], 2)$ left medial orbitofrontal

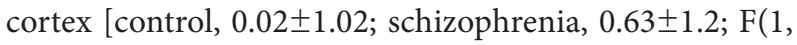
$183)=13.95, \mathrm{p}<0.001], 3)$ left middle temporal gyrus [control, $0.02 \pm 1.03$; schizophrenia, 0.58 $\pm 1.06 ; \mathrm{F}(1,183)=14.82, \mathrm{p}<$ 0.001 ] 4) left pars opercularis [control, $0.05 \pm 1.01$; schizophrenia, $0.68 \pm 1.1 ; \mathrm{F}(1,183)=17.45, \mathrm{p}<0.001]$, 5) left rostral anterior cingulate cortex [control, $0.04 \pm 1.04$; schizophrenia, $0.48 \pm 1.09 ; \mathrm{F}(1,183)=8.36, \mathrm{p}=0.004], 6)$ left rostral middle frontal gyrus [control, $0.04 \pm 1.01$; schizophrenia, $0.45 \pm 0.89$; $\mathrm{F}(1,183)=9.11, \mathrm{p}=0.002], 7)$ left STG [control, $0.06 \pm 1.01$; schizophrenia, $0.59 \pm 1.02 ; \mathrm{F}(1,183)=14.27, \mathrm{p}<0.001]$, 8) left temporal pole [control, $0.01 \pm 1.01$; schizophrenia, $0.47 \pm 1.22$; $\mathrm{F}(1,183)=7.8, \mathrm{p}=0.005$ ], and 9) right frontal pole [control, $0.01 \pm$ 0.5 ; schizophrenia, $1.03 \pm 1.23 ; \mathrm{F}(1,183)=9.15, \mathrm{p}=0.002]$.

\section{Relationship between GM diffusivity and working memory}

The correlation results are displayed in Table 3. In the patient group, we evaluated the Pearson partial correlations between the LNS scaled scores and the diffusivities (AD, RD, and TR) of the ROIs with diffusivity values that differed significantly from those of the control group. The $\mathrm{AD}$ values were
Table 3. Correlations between the LNS scores and diffusivity values in the ROIs with significant differences between the groups

\begin{tabular}{llcc}
\hline & \multicolumn{1}{c}{ ROI in schizophrenia } & $\mathrm{r}$ & $\mathrm{p}$ \\
\hline $\mathrm{AD}$ & Left lateral orbitofrontal cortex & -0.23 & 0.03 \\
& Left superior temporal gyrus & -0.24 & 0.02 \\
$\mathrm{RD}$ & Left inferior temporal gyrus & -0.21 & 0.05 \\
& Left lateral orbitofrontal cortex & -0.25 & 0.02 \\
$\mathrm{~T}$ & Left lateral orbitofrontal cortex & -0.27 & 0.01 \\
& Left rostral anterior cingulate cortex & -0.21 & 0.04 \\
& Left superior temporal gyrus & -0.22 & 0.04 \\
\hline
\end{tabular}

LNS: letter number sequencing, ROI: region of interest, AD: axial diffusivity, RD: radial diffusivity, TR: trace

significantly and negatively correlated with the LNS scores in the following ROIs (correlation coefficients, uncorrected $\mathrm{p}$ value) (Figure 1): 1) Left lateral orbitofrontal cortex ( $r=-0.23$, $\mathrm{p}=0.03)$ and 2) Left STG $(\mathrm{r}=-0.24, \mathrm{p}=0.02)$. The RD values significantly and negatively correlated with the LNS scores in the following ROIs (Figure 2): 1) Left inferior temporal gyrus $(\mathrm{r}=-0.21, \mathrm{p}=0.05)$ and 2$)$ Left lateral orbitofrontal cortex $(\mathrm{r}=-$ $0.25, \mathrm{p}=0.02$ ). The TR values were significantly and negatively correlated with the LNS scores in the following ROIs (Figure 3): 1) left lateral orbitofrontal cortex ( $r=-0.27, p=0.01), 2)$ left rostral anterior cingulate cortex $(\mathrm{r}=-0.21, \mathrm{p}=0.04)$, and 3$)$ left STG $(r=-0.22, \mathrm{p}=0.04)$.

\section{DISCUSSION}

The purpose of this study was to compare the GM microstructures between the schizophrenia and control groups and investigate the relationship between GM diffusivity and working memory. Compared with the control group, the schizophrenia group showed significantly higher $A D, R D$, and TR values in specific regions in frontal, temporal, and anterior cingulate regions. Working memory and diffusivity $(\mathrm{AD}, \mathrm{RD}$, and $\mathrm{TR})$ were negatively correlated in the lateral orbitofrontal, superior temporal, inferior temporal, and rostral anterior cingulate areas of the left hemisphere in the individuals with schizophrenia.

Previous GM-DTI studies have shown that, compared with controls, individuals with schizophrenia have increased $\mathrm{AD}$ and $\mathrm{RD}$ values in the prefrontal cortex, ${ }^{13}$ increased $\mathrm{AD}$, $\mathrm{RD}$, and TR values in the left bank of the superior temporal sulcus; ${ }^{11}$ and increased MD, similar to TR, in the STG. ${ }^{12}$ Our results were partly consistent with the previous results. Increased RD and TR values indicate microstructural abnormalities that suggest alterations in intrinsic connections, ${ }^{52} \mathrm{a}$ loss of dendritic spines, ${ }^{38,53,54}$ and/or increased volume of extracellular water due to inflammation. ${ }^{55-57}$ No significant changes were found in the FA but were found in the TR because FA 

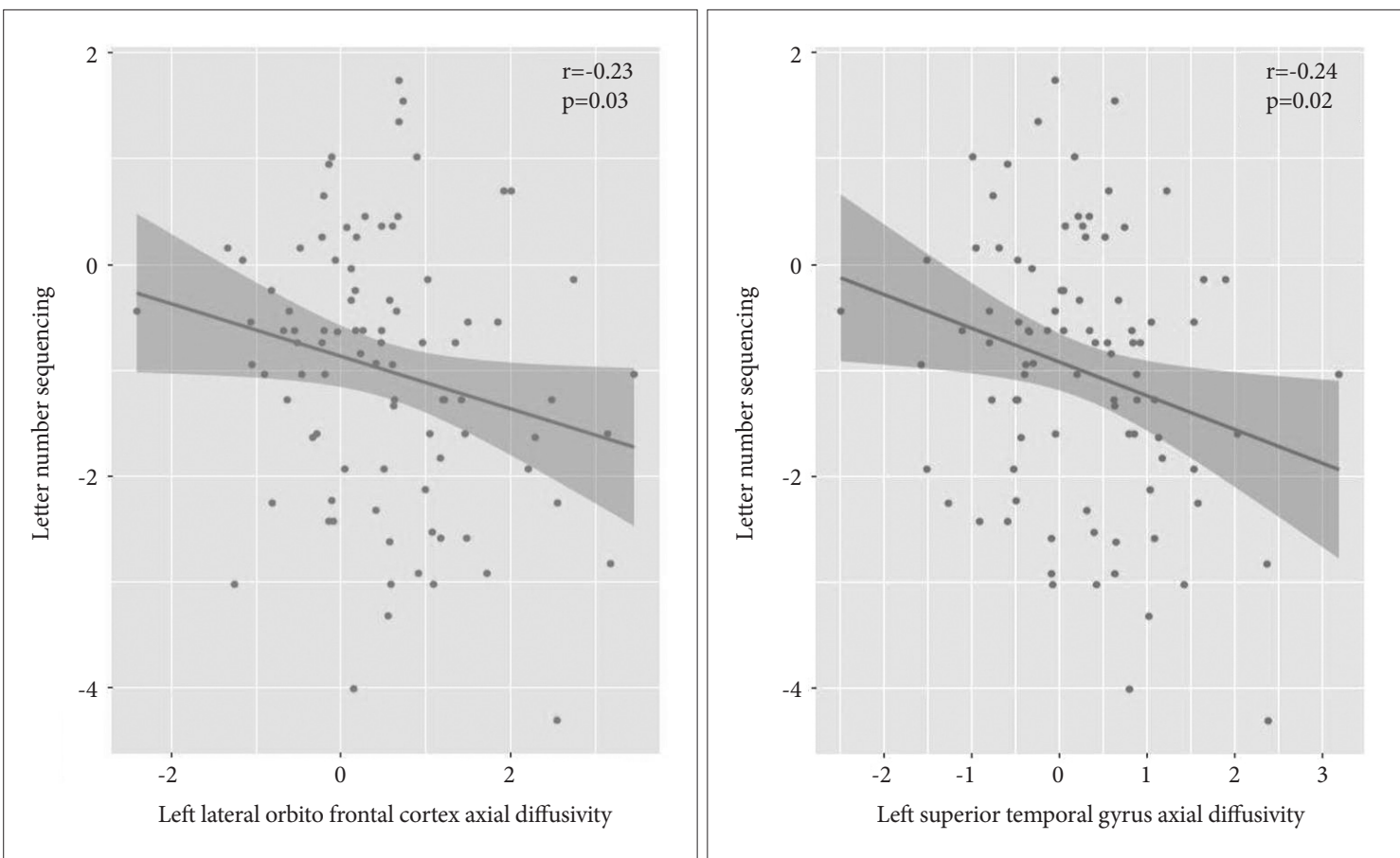

Figure 1. Relationship between the axial diffusivity values and letter number sequencing scores in the left lateral orbitofrontal cortex and superior temporal gyrus in individuals with schizophrenia.
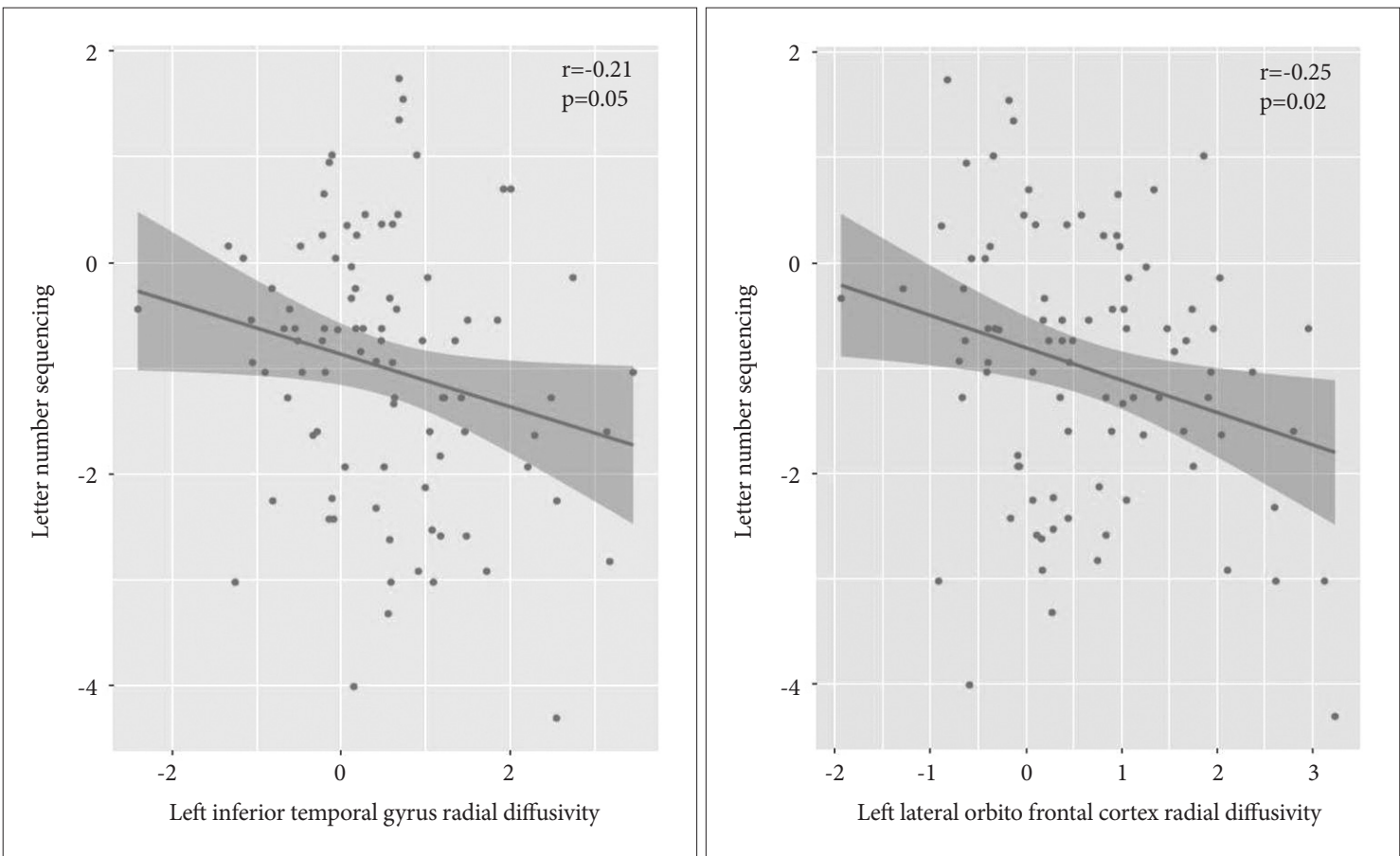

Figure 2. Relationship between the radial diffusivity values and letter number sequencing scores in the left lateral orbitofrontal cortex and inferior temporal gyrus in individuals with schizophrenia.

measures directionality and TR measures the proportion of water movement. Thus, TR, and FA can be independent of each other. ${ }^{58}$ Therefore, the findings of increased TR and no change in FA might be associated with increased water con- tent as in cases of edema ${ }^{59}$ or a subtle loss of neurological processes. ${ }^{39,52,60}$ Interestingly, the AD values were increased in the schizophrenia group, which differed from the findings of previous DTI studies on schizophrenia. However, several 

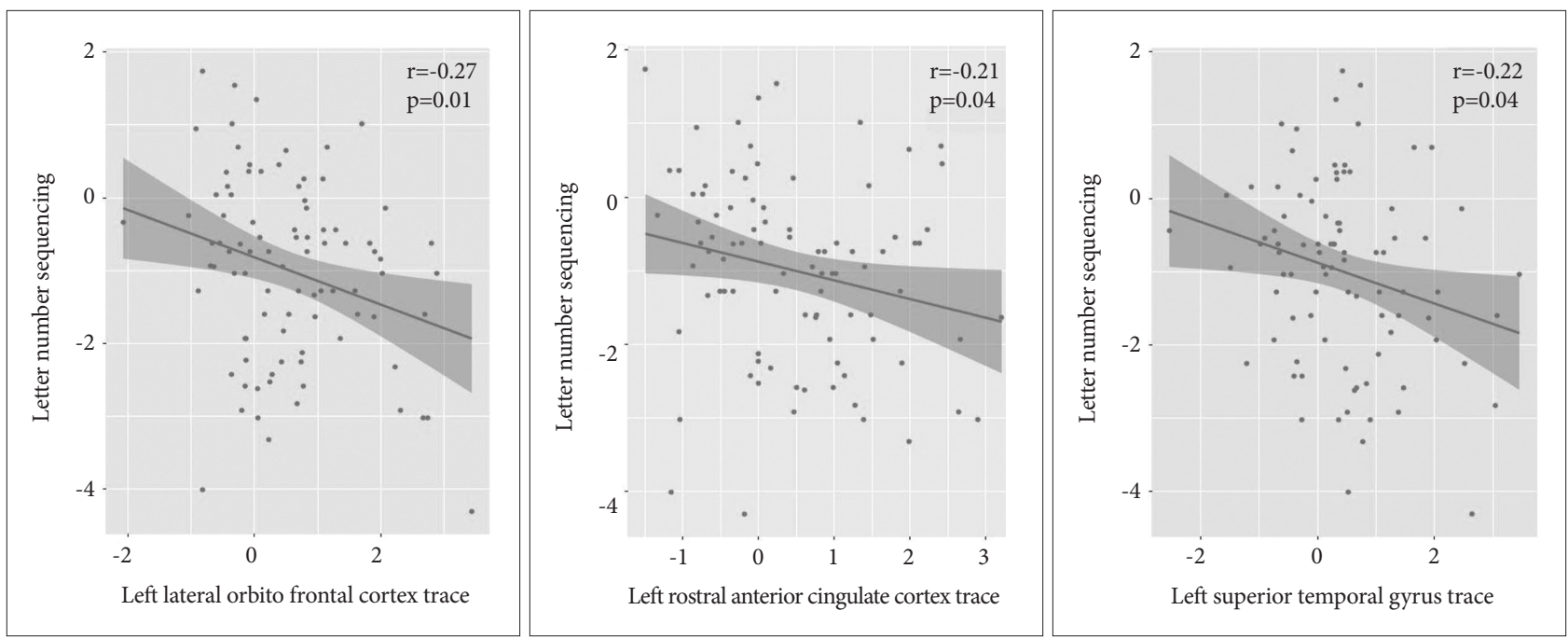

Figure 3. Relationship between the trace diffusivity values and letter number sequencing scores in the left lateral orbitofrontal cortex, superior temporal gyrus, and rostral anterior cingulate cortex in individuals with schizophrenia.

DTI-schizophrenia studies have reported increased AD values and suggested that decreases or delays in the normal pruning process or neurodegenerative processes may lead to axonal loss $^{61}$ and increased water content outside of the axonal space. ${ }^{62}$ These findings suggest that increased $\mathrm{AD}$ is associated with neurological issues.

The previous studies investigating the association between the brain structure and working memory have deemed that the orbitofrontal cortex is necessary for the working memory maintenance, manipulation, and monitoring processes, and high levels of cognitive load. ${ }^{63,64}$ LNS is an appropriate test of such conditions. LNS requires the ability to monitor a series of complex stimuli, maintain recently processed the items, and manipulates items in a series to identify correct sorting. The results of our study support those of a previous study that reported an association between the orbitofrontal cortex and performance on working memory tasks requiring high levels of cognitive load, such as the LNS. Thus, considering our results were indicative of increased diffusivity (RD and TR) in the orbitofrontal cortex, microstructural abnormalities in the orbitofrontal cortex might serve as an indicator of the processing of the coordination of the working memory maintenance, manipulation, and monitoring processes.

Abnormalities in the anterior cingulate cortex, which is a key component of working memory, are strongly associated with schizophrenia. ${ }^{65-67}$ Several studies have reported that abnormal synaptic connectivity and decreased neuronal density in the anterior cingulate cortex may contribute to working memory impairments in schizophrenia. ${ }^{68-71}$ In addition, the rostral and caudal anterior cingulate cortices share inputs with a network involving associative, limbic, sensorimotor, dorsolateral prefrontal area, which is a structure that is inde- pendently linked to working memory. ${ }^{72}$ We found negative correlations between working memory and diffusivity, indicating microstructural abnormalities in the anterior cingulate cortex. Based on previous findings, our results suggest that working memory problems are related to difficulty in the sharing of information with each area that is connected to the anterior cingulate cortex due to microstructural abnormalities, such as synaptic dysconnectivity and decreased neuronal density in the anterior cingulate cortex.

Temporal areas are consistently activated during working memory performance. ${ }^{73}$ In addition, the left inferior temporal and left superior temporal gyri are associated with shortterm memory. ${ }^{74,75}$ Previous studies with patients with shortterm memory impairments have reported that decreased verbal working memory is due to damage to verbal shortterm. ${ }^{76-78}$ Considering these previous results and our results of the negative correlation between working memory and diffusivity in temporal areas, we suggest that microstructural deficits in temporal areas result in poor working performance and are therefore associated with short-term memory deficits for working memory load.

The strength of this study is that we studied the whole GM region with more than 150 participants using a large database. According to previous schizophrenia studies, there are more than 50 regional deficits, such as frontal, temporal, parietal, cingulate area and cuneus deficits, ${ }^{41}$ and a larger group size will give us the specificity of microscopic alterations and the implication that the alterations are associated with symptoms, or clinical profiles. ${ }^{42}$ Previous GM-DTI studies were conducted within a specific GM region or small sample size. Such studies may ignore GM regions that are important from a clinical perspective or require more explanatory power. 
Therefore, a GM-DTI study of the whole GM region with a larger sample size is meaningful to increase the explanatory power of GM microstructural abnormalities.

Our study had the following limitations. First, we did not measure other cognitive functions because only the scale scores for the LNS test were available in the datasets of the two different research projects. Future studies should investigate the associations of other cognitive functions with GM diffusivity. Second, this study used data from two different projects. Thus, our study was conducted on data that were obtained from different neuroimaging systems and different cognitive batteries. Therefore, standard scores were used within the framework of our study. Future studies need to be conducted using the same protocol and similar sample sizes. Third, we did not consider illness duration or the duration of medications in this study because the two research projects did not contain the same clinical data. Future studies need to be conducted for comparisons to the results of this study, especially in consideration of these variables.

Our results indicated GM microstructural abnormalities in the frontal, temporal, and anterior cingulate regions of individuals with schizophrenia. Furthermore, these regional GM microstructural abnormalities suggest a neuropathological basis for the working memory deficits clinically observed in individuals with schizophrenia.

\section{Supplementary Materials}

The online-only Data Supplement is available with this article at https://doi.org/10.30773/pi.2018.10.14.1.

\section{Acknowledgments}

The data were downloaded using the Collaborative Informatics and Neuroimaging Suite Data Exchange tool (COINS; http://coins.mrn.org/ $\mathrm{dx})$. The data collection performed at the Mind Research Network was funded by NIH grant 5P20RR021938/P20GM103472 to Dr. Vince Calhoun of the Center of Biomedical Research Excellence (COBRE).

The data used in this study were obtained from the Neuromorphometry by Computer Algorithm Chicago (NMorphCH) dataset (http://nunda. northwestern.edu/nunda/data/projects/NMorphCHThe data collection and sharing of the NMorphCH project were funded by NIMH grant R01 MH056584.

The data used in this study were obtained from the SchizConnect database (http://schizconnect.org). The data collection and sharing of the SchizConnect project were funded by NIMH cooperative agreement $1 \mathrm{U} 01$ MH097435.

This paper was based on the first author's thesis from Chung-Ang University.

\section{REFERENCES}

1. Lett TA, Voineskos AN, Kennedy JL, Levine B, Daskalakis ZJ. Treating working memory deficits in schizophrenia: a review of the neurobiology. Biol Psychiatry 2014;75:361-370.

2. Green MF. What are the functional consequences of neurocognitive deficits in schizophrenia? Am J Psychiatry 1996;153:321-330.

3. Dazzan P, Arango C, Fleischacker W, Galderisi S, Glenthøj B, Leucht S, et al. Magnetic resonance imaging and the prediction of outcome in first-episode schizophrenia: a review of current evidence and directions for future research. Schizophr Bull 2015;41:574-583.

4. Karlsgodt KH, Niendam TA, Bearden CE, Cannon TD. White matter integrity and prediction of social and role functioning in subjects at ultra-high risk for psychosis. Biol Psychiatry 2009;66:562-569.

5. Karlsgodt KH, Kochunov P, Winkler AM, Laird AR, Almasy L, Duggirala R, et al. A multimodal assessment of the genetic control over working memory. J Neurosci 2010;30:8197-8202.

6. Nazeri A, Chakravarty MM, Felsky D, Lobaugh NJ, Rajji TK, Mulsant $\mathrm{BH}$, et al. Alterations of superficial white matter in schizophrenia and relationship to cognitive performance. Neuropsychopharmacology 2013;38:1954-1962.

7. Bartzokis G, Lu PH, Tingus K, Mendez MF, Richard A, Peters DG, et al. Lifespan trajectory of myelin integrity and maximum motor speed. Neurobiol Aging 2010;31:1554-1562.

8. Bartzokis G. Neuroglialpharmacology: myelination as a shared mechanism of action of psychotropic treatments. Neuropharmacology 2012;62:2137-2153.

9. Felts PA, Baker TA, Smith KJ. Conduction in segmentally demyelinated mammalian central axons. J Neurosci 1997;17:7267-7277.

10. Waxman S, Bennett M. Relative conduction velocities of small myelinated and non-myelinated fibres in the central nervous system. Nat New Biol 1972;238:217-219.

11. Lee JS, Kim CY, Joo YH, Newell D, Bouix S, Shenton ME, et al. Increased diffusivity in gray matter in recent onset schizophrenia is associated with clinical symptoms and social cognition. Schizophr Res 2016;176:144-150

12. Lee K, Yoshida T, Kubicki M, Bouix S, Westin CF, Kindlmann G, et al. Increased diffusivity in superior temporal gyrus in patients with schizophrenia: a diffusion tensor imaging study. Schizophr Res 2009;108:33-40.

13. Park JY, Park HJ, Kim DJ, Kim JJ. Positive symptoms and water diffusivity of the prefrontal and temporal cortices in schizophrenia patients: a pilot study. Psychiatry Res 2014;224:49-57.

14. Shin YW, Kwon JS, Ha TH, Park HJ, Kim DJ, Hong SB, et al. Increased water diffusivity in the frontal and temporal cortices of schizophrenic patients. Neuroimage 2006;30:1285-1291.

15. Kalus P, Slotboom J, Gallinat J, Federspiel A, Gralla J, Remonda L, et al. New evidence for involvement of the entorhinal region in schizophrenia: a combined MRI volumetric and DTI study. Neuroimage 2005;24: 1122-1129.

16. Moriya J, Kakeda S, Abe O, Goto N, Yoshimura R, Hori H, et al. Gray and white matter volumetric and diffusion tensor imaging (DTI) analyses in the early stage of first-episode schizophrenia. Schizophr Res 2010;116:196-203.

17. Kroenke CD, Van Essen DC, Inder TE, Rees S, Bretthorst GL, Neil JJ, et al. Microstructural changes of the baboon cerebral cortex during gestational development reflected in magnetic resonance imaging diffusion anisotropy. J Neurosci 2007;27:12506-12515.

18. Bock AS, Olavarria JF, Leigland LA, Taber EN, Jespersen SN, Kroenke CD. Diffusion tensor imaging detects early cerebral cortex abnormalities in neuronal architecture induced by bilateral neonatal enucleation: an experimental model in the ferret. Front Syst Neurosci 2010;4:149.

19. Thornton JS, Ordidge RJ, Penrice J, Cady EB, Amess PN, Punwani S, et al. Anisotropic water diffusion in white and gray matter of the neonatal piglet brain before and after transient hypoxia-ischaemia. Magn Reson Imaging 1997;15:433-440.

20. Sizonenko SV, Camm EJ, Garbow JR, Maier SE, Inder TE, Williams $\mathrm{CE}$, et al. Developmental changes and injury induced disruption of the radial organization of the cortex in the immature rat brain revealed by in vivo diffusion tensor MRI. Cereb Cortex 2007;17: 2609-2617.

21. Gupta RK, Hasan KM, Trivedi R, Pradhan M, Das V, Parikh NA, et al. Diffusion tensor imaging of the developing human cerebrum. J Neurosci Res 2005;81:172-178. 
22. Trivedi R, Gupta RK, Husain N, Rathore RK, Saksena S, Srivastava S, et al. Region-specific maturation of cerebral cortex in human fetal brain: diffusion tensor imaging and histology. Neuroradiology 2009; 51:567-576.

23. McKinstry RC, Mathur A, Miller JH, Ozcan A, Snyder AZ, Schefft GL, et al. Radial organization of developing preterm human cerebral cortex revealed by non-invasive water diffusion anisotropy MRI. Cereb Cortex 2002;12:1237-1243.

24. Maas LC, Mukherjee P, Carballido-Gamio J, Veeraraghavan S, Miller SP, Partridge SC, et al. Early laminar organization of the human cerebrum demonstrated with diffusion tensor imaging in extremely premature infants. NeuroImage 2004;22:1134-1140.

25. DeIpolyi AR, Mukherjee P, Gill K, Henry RG, Partridge SC, Veeraraghavan $\mathrm{S}$, et al. Comparing microstructural and macrostructural development of the cerebral cortex in premature newborns: diffusion tensor imaging versus cortical gyration. NeuroImage 2005;27: 579586.

26. Mukherjee P, McKinstry RC. Diffusion tensor imaging and tractography of human brain development. Neuroimaging Clin N Am 2006;16: $19-43$.

27. Dyrby TB, Baaré WFC, Alexander DC, Jelsing J, Garde E, Søgaard LV. An ex vivo imaging pipeline for producing high- quality and high-resolution diffusion-weighted imaging datasets. Hum Brain Mapp 2011; 32:544-563.

28. McNab JA, Polimeni JR, Wang R, Augustinack JC, Fujimoto K, Player A, et al. Surface based analysis of diffusion orientation for identifying architectonic domains in the in vivo human cortex. NeuroImage 2013; 69:87-100

29. McNab JA, Jbabdi S, Deoni SC, Douaud G, Behrens TE, Miller KL. High resolution diffusion-weighted imaging in fixed human brain using diffusion-weighted steady state free precession. Neuroimage 2009; 46:775-785.

30. Miller KL, Stagg CJ, Douaud G, Jbabdi S, Smith SM, Behrens TE, et al. Diffusion imaging of whole, post-mortem human brains on a clinical MRI scanner. Neuroimage 2011;57:167-181.

31. Jaermann T, De Zanche N, Staempfli P, Pruessmann KP, Valavanis A, Boesiger $\mathrm{P}$, et al. Preliminary experience with visualization of intracortical fibers by focused high-resolution diffusion tensor imaging. AJNR Am J Neuroradiol 2008;29:146-150.

32. Heidemann RM, Anwander A, Feiweier T, Knösche TR, Turner R. kspace and q-space: combining ultra-high spatial and angular resolution in diffusion imaging using ZOOPPA at 7 T. Neuroimage 2012;60: 967-978.

33. Heidemann RM, Porter DA, Anwander A, Feiweier T, Heberlein K, Knösche TR, et al. Diffusion imaging in humans at 7T using readoutsegmented EPI and GRAPPA. Magn Reson Med 2010;64:9-14.

34. Truong TK, Guidon A. High-resolution multishot spiral diffusion tensor imaging with inherent correction of motion-induced phase errors. Magn Reson Med 2014;71:790-796.

35. Sotiropoulos SN, Jbabdi S, Xu J, Andersson JL, Moeller S, Auerbach $\mathrm{EJ}$, et al. Advances in diffusion MRI acquisition and processing in the Human Connectome Project. Neuroimage 2013;80:125-143.

36. Golay X, Jiang H, vanZijl P, Mori S. High-resolution isotropic 3D diffusion tensor imaging of the human brain. Magn Reson Med 2002;47:837-843.

37. Briggs F. Organizing principles of cortical layer 6. Front Neural Circuits 2010;4:1-8.

38. Bennett M. Schizophrenia: susceptibility genes, dendritic-spine pathology and gray matter loss. Prog Neurobiol 2011;95:275-300.

39. Costa E, Davis J, Grayson DR, Guidotti A, Pappas GD, Pesold C. Dendritic spine hypoplasticity and downregulation of reelin and GABAergic tone in schizophrenia vulnerability. Neurobiol Dis 2001;8:723-742.

40. Glantz LA, Lewis DA. Decreased dendritic spine density on prefrontal cortical pyramidal neurons in schizophrenia. Arch Gen Psychiatry 2000;57:65-73.
41. Honea R, Crow TJ, Passingham D, Mackay CE. Regional deficits in brain volume in schizophrenia: a meta-analysis of voxel-based morphometry studies. Am J Psychiatry 2005;162:2233-2245.

42. Kubicki M, Shenton ME. Diffusion tensor imaging findings and their implications in schizophrenia. Curr Opin Psychiatry 2014;27:179-184.

43. Wang L, Alpert KI, Calhoun VD, Cobia DJ, Keator DB, King MD, et al. SchizConnect: mediating neuroimaging databases on schizophrenia and related disorders for large-scale integration. Neuroimage 2016;124:1155-1167.

44. Çetin MS, Christensen F, Abbott CC, Stephen JM, Mayer AR, Cañive JM, et al. Thalamus and posterior temporal lobe show greater internetwork connectivity at rest and across sensory paradigms in schizophrenia. Neuroimage 2014;97:117-126.

45. Jenkinson M, Smith S. A global optimisation method for robust affine registration of brain images. Med Image Anal 2001;5:143-156.

46. Jenkinson M, Bannister P, Brady M, Smith S. Improved optimization for the robust and accurate linear registration and motion correction of brain images. Neuroimage 2002;17:825-841.

47. Fischl B, Salat DH, Busa E, Albert M, Dieterich M, Haselgrove C, et al. Whole brain segmentation: automated labeling of neuroanatomical structures in the human brain. Neuron 2002;33:341-355.

48. Kern RS, Nuechterlein KH, Green MF, Baade LE, Fenton WS, Gold JM, et al. The MATRICS Consensus Cognitive Battery, part 2: conorming and standardization. Am J Psychiatry 2008;165:214-220.

49. Nuechterlein KH, Green MF, Kern RS, Baade LE, Barch DM, Cohen JD, et al. The MATRICS Consensus Cognitive Battery, part 1: test selection, reliability, and validity. Am J Psychiatry 2008;165:203-213.

50. Wechsler D. Wechsler Memory Scale (WMS-III). San Antonio, TX; Psychological Corporation:1997.

51. R Core Team. R: A Language and Environment for Statistical Computing. Vienna: R Foundation for Statistical Computing; 2017.

52. Lewis DA, Gonzalez-Burgos G. Intrinsic excitatory connections in the prefrontal cortex and the pathophysiology of schizophrenia. Brain Res Bull 2000;52:309-317.

53. Glausier JR, Lewis DA. Dendritic spine pathology in schizophrenia. Neuroscience 2013;251:90-107.

54. Konopaske GT, Lange N, Coyle JT, Benes FM. Prefrontal cortical dendritic spine pathology in schizophrenia and bipolar disorder. JAMA Psychiatry 2014;71:1323-1331.

55. Doorduin J, De Vries EF, Willemsen AT, De Groot JC, Dierckx RA, Klein HC. Neuroinflammation in schizophrenia-related psychosis: a PET study. J Nucl Med 2009;50:1801-1807.

56. Pasternak O, Westin CF, Bouix S, Seidman LJ, Goldstein JM, Woo TU, et al. Excessive extracellular volume reveals a neurodegenerative pattern in schizophrenia onset. J Neurosci 2012;32:17365-17372.

57. 57. Potvin S, Stip E, Sepehry AA, Gendron A, Bah R, Kouassi E. Inflammatory cytokine alterations in schizophrenia: a systematic quantitative review. Biol Psychiatry 2008;63:801-808.

58. 58. Wieshmann UC, Clark CA, Symms MR, Franconi F, Barker GJ, Shorvon SD. Reduced anisotropy of water diffusion in structural cerebral abnormalities demonstrated with diffusion tensor imaging. Magn Reson Imaging 1999;17:1269-1274.

59. Alexander AL, Lee JE, Lazar M, Field AS. Diffusion tensor imaging of the brain. Neurotherapeutics 2007;4:316-329.

60. Glantz LA, Lewis DA. Decreased dendritic spine density on prefrontal cortical pyramidal neurons in schizophrenia. Arch Gen Psychiatry 2000;57:65-73.

61. Clark K, Narr KL, O’Neill J, Levitt J, Siddarth P, Phillips O, et al. White matter integrity, language, and childhood onset schizophrenia. Schizophr Res 2012;138:150-156.

62. Lu LH, Zhou XJ, Keedy SK, Reilly JL, Sweeney JA. White matter microstructure in untreated first episode bipolar disorder with psychosis: comparison with schizophrenia. Bipolar Disord 2011;13:604-613.

63. Barbey AK, Koenigs M, Grafman J. Orbitofrontal contributions to human working memory. Cereb Cortex 2010;21:789-795. 
64. Lara AH, Kennerley SW, Wallis JD. Encoding of gustatory working memory by orbitofrontal neurons. J Neurosci 2009;29:765-774.

65. Bush G, Luu P, Posner MI. Cognitive and emotional influences in anterior cingulate cortex. Trends Cogn Sci 2000;4:215-222.

66. Fornito A, Yücel M, Patti J, Wood S, Pantelis C. Mapping grey matter reductions in schizophrenia: an anatomical likelihood estimation analysis of voxel-based morphometry studies. Schizophr Res 2009;108:104-113.

67. Minzenberg MJ, Laird AR, Thelen S, Carter CS, Glahn DC. Metaanalysis of 41 functional neuroimaging studies of executive function in schizophrenia. Arch Gen Psychiatry 2009;66:811-822.

68. Bouras C, Kövari E, Hof PR, Riederer BM, Giannakopoulos P. Anterior cingulate cortex pathology in schizophrenia and bipolar disorder. Acta Neuropathol 2001;102:373-379.

69. Broadbelt K, Byne W, Jones LB. Evidence for a decrease in basilar dendrites of pyramidal cells in schizophrenic medial prefrontal cortex. Schizophr Res 2002;58:75-81.

70. Kreczmanski P, Schmidt-Kastner R, Heinsen H, Steinbusch HW, Hof PR, Schmitz C. Stereological studies of capillary length density in the frontal cortex of schizophrenics. Acta Neuropathol 2005;109:510-518.

71. Todtenkopf MS, Vincent SL, Benes FM. A cross-study meta-analysis and three-dimensional comparison of cell counting in the anterior cingulate cortex of schizophrenic and bipolar brain. Schizophr Res 2005;73:79-89.

72. Habas C. Functional connectivity of the human rostral and caudal cingulate motor areas in the brain resting state at 3T. Neuroradiology 2010;52:47-59.

73. Rottschy C, Langner R, Dogan I, Reetz K, Laird AR, Schulz JB, et al. Modelling neural correlates of working memory: a coordinate-based meta-analysis. Neuroimage 2012;60:830-846.

74. Leff AP, Schofield TM, Crinion JT, Seghier ML, Grogan A, Green DW, et al. The left superior temporal gyrus is a shared substrate for auditory short-term memory and speech comprehension: evidence from 210 patients with stroke. Brain 2009;132:3401-3410.

75. Smith EE, Jonides J. Working Memory in Humans: Neuropsychological Evidence. Cambridge: The MIT Press; 1995.

76. Shallice T, Butterworth B. Short-term memory impairment and spontaneous speech. Neuropsychologia 1977;15:729-735.

77. Unsworth N, Engle RW. On the division of short-term and working memory: an examination of simple and complex span and their relation to higher order abilities. Psychol Bull 2007;133:1038-1066.

78. Vallar G, Baddeley AD. Fractionation of working memory: neuropsychological evidence for a phonological short-term store. J Verb Learn Verb Behav 1984;23:151-161. 


\begin{tabular}{|c|c|c|c|}
\hline \multicolumn{4}{|c|}{ Regions of interest } \\
\hline \multicolumn{3}{|c|}{ Left hemisphere } & \multirow{2}{*}{$\begin{array}{c}\text { Right hemisphere } \\
\text { Right bank of the superior temporal sulcus }\end{array}$} \\
\hline 1 & Left bank of the superior temporal sulcus & 35 & \\
\hline 2 & Left caudal anterior cingulate cortex & 36 & Right caudal anterior cingulate cortex \\
\hline 3 & Left caudal middle frontal gyrus & 37 & Right caudal middle frontal gyrus \\
\hline 4 & Left cuneus & 38 & Right cuneus \\
\hline 5 & Left entorhinal cortex & 39 & Right entorhinal cortex \\
\hline 6 & Left frontal pole & 40 & Right frontal pole \\
\hline 7 & Left fusiform gyrus & 41 & Right fusiform gyrus \\
\hline 8 & Left inferior parietal cortex & 42 & Right inferior parietal cortex \\
\hline 9 & Left inferior temporal gyrus & 43 & Right inferior temporal gyrus \\
\hline 10 & Left insula cortex & 44 & Right insula cortex \\
\hline 11 & Left isthmus of the cingulate gyrus & 45 & Right isthmus of the cingulate gyrus \\
\hline 12 & Left lateral occipital cortex & 46 & Right lateral occipital cortex \\
\hline 13 & Left lateral orbitofrontal cortex & 47 & Right lateral orbitofrontal cortex \\
\hline 14 & Left lingual gyrus & 48 & Right lingual gyrus \\
\hline 15 & Left medial orbitofrontal cortex & 49 & Right medial orbitofrontal cortex \\
\hline 16 & Left middle temporal gyrus & 50 & Right middle temporal gyrus \\
\hline 17 & Left paracentral lobule & 51 & Right paracentral lobule \\
\hline 18 & Left parahippocampal gyrus & 52 & Right parahippocampal gyrus \\
\hline 19 & Left pars opercularis & 53 & Right pars opercularis \\
\hline 20 & Left pars orbitalis & 54 & Right pars orbitalis \\
\hline 21 & Left pars triangularis & 55 & Right pars triangularis \\
\hline 22 & Left pericalcarine & 56 & Right pericalcarine \\
\hline 23 & Left postcentral cortex & 57 & Right postcentral cortex \\
\hline 24 & Left posterior cingulate cortex & 58 & Right posterior cingulate cortex \\
\hline 25 & Left precentral cortex & 59 & Right precentral cortex \\
\hline 26 & Left precuneus & 60 & Right precuneus \\
\hline 27 & Left rostral anterior cingulate cortex & 61 & Right rostral anterior cingulate cortex \\
\hline 28 & Left rostral middle frontal gyrus & 62 & Right rostral middle frontal gyrus \\
\hline 29 & Left superior frontal gyrus & 63 & Right superior frontal gyrus \\
\hline 30 & Left superior parietal cortex & 64 & Right superior parietal cortex \\
\hline 31 & Left superior temporal gyrus & 65 & Right superior temporal gyrus \\
\hline 32 & Left supramarginal gyrus & 66 & Right supramarginal gyrus \\
\hline 33 & Left temporal pole & 67 & Right temporal pole \\
\hline 34 & Left transverse temporal gyrus & 68 & Right transverse temporal gyrus \\
\hline
\end{tabular}

